\title{
Plasmon absorption in nanospheres: a comparison of sodium, potassium, aluminium, silver and gold
}

\author{
M.G. Blaber ${ }^{1}$, M.D. Arnold ${ }^{2}$, N. Harris ${ }^{1}$, M.J Ford ${ }^{1}$ and M.B. Cortie $^{1}$ \\ 1 Institute for Nanoscale Technology, University of Technology Sydney, Sydney, NSW, Australia, \\ 2 MacDiarmid Institute and Dept. of Electrical and Computer Engineering, University of \\ Canterbury, Private Bag 4800, Christchurch, New Zealand \\ *To whom correspondance should be addressed:mike.ford@uts,edu.au
}

\begin{abstract}
The optical absorption for nanospheres made from $\mathrm{Na}, \mathrm{K}, \mathrm{Al}, \mathrm{Ag}$ and $\mathrm{Au}$ are compared as a precursor to choosing the ideal metal for use in a negative permittivity (NP) near-field superlens. The relationship between optical absorption of the metal nanosphere and the ability of the NP lens to reconstruct the near-field is one to one. Metals with low dielectric losses have large plasmon absorption cross-sections and absorb over a very narrow wavelength range; they are consequently excellent materials for superlenses. Numerical solutions to Mie theory were used to calculate the absorption efficiency, $Q_{a b s}$, for nanospheres varying in radius between $5 \mathrm{~nm}$ and $100 \mathrm{~nm}$ in vacuum. We show that, although silver is the most commonly used material for superlensing, its absorption efficiency, as a nanosphere, at the plasmon resonance is not as strong as materials such as the alkali metals. Of all these materials, potassium spheres with a radius of $21 \mathrm{~nm}$ have an optimum absorption efficiency of 14.7, resulting in the ability of a film with thickness of $40 \mathrm{~nm}$ to reconstruct a grating with a period of $57 \mathrm{~nm}$.
\end{abstract}

Keywords : sodium, potassium, aluminium, silver and gold-nanoparticles, plasmon resonance, superlens, nanolithography

\section{Introduction}

The focus of most research on the optical properties of metal nanoparticles has been absorption and scattering due to plasmon resonance, directly resulting in applications in energy efficient coatings ${ }^{1}$, cancer therapy ${ }^{2-4}$ and lithography $^{5}$ to name a few. The optical properties of nanoparticles depend greatly on size, shape, surrounding medium and dielectric constant. ${ }^{6}$ Work on the optimization of plasmonic heating in nanoparticles based on size and shape is well established. ${ }^{7}$ Here, focus is placed on the absorption efficiency of spheres as a function of size and element. The relationship between the optical properties of metallic nanospheres and the imaging properties of NP lenses remains hitherto unexplored. The ability of a metal sphere to absorb is not often described in the literature as an absence of losses in the permittivity of the metal. In section 2 we briefly describe the optical absorption of metal spheres with radii between $5 \mathrm{~nm}$ and $50 \mathrm{~nm}$ in terms of bulk dielectric constants.

Veselago showed in 1968 that materials with negative permeability and negative permittivity would have a negative refractive index. ${ }^{8}$ In 2000 , Pendry proposed that a lens made of a thin film of silver could reconstruct p-polarized fields and subvert the diffraction limit due to the negative permittivity of the silver. ${ }^{9}$ Since then, many groups have worked on the use of silver as a NP superlens with its main application in nanoscale lithography at optical wavelengths. ${ }^{10-14}$ Unfortunately, problems with losses in the silver ${ }^{15}$ and bandwidth restrictions result in an image with finite resolution. In section 3 we propose the use of thin potassium films that can provide greater resolution.

\section{Absorption in noble and alkali metal nanospheres}

Bulk dielectric constants from Weaver and Frederikse $^{16}$ along with a numerical implementation of Mie theory ${ }^{17}$ named 'BHCOAT' ${ }^{\text {' }}$ were used to calculate the absorption and scattering efficiencies $\left(Q_{a b s}\right.$ and $Q_{\text {sca }}$, respectively) of metal nanospheres with radii between $5 \mathrm{~nm}$ and $50 \mathrm{~nm}$. The particle size range was chosen such that the absorption spectra were dominated by the dipole plasmon resonance. Sphere size was optimized to give the highest possible absorption efficiency at 
any wavelength. The sphere radius and wavelength for which maximum absorption efficiency occurs is shown in Table 1 alongside the permittivity of the metal at that wavelength. Fig 1 shows the spectra corresponding to the sphere radius that causes maximum $Q_{a b s}$. The absorption peaks for $\mathrm{K}, \mathrm{Al}$ and $\mathrm{Na}$ are sharp with $Q_{a b s}$ equal to 14.7, 13.0 and 12.4, respectively. These values are significantly higher than those for both $\mathrm{Ag}$ and $\mathrm{Au}$ which have maximum $Q_{a b s}$ of 5.8 and 3.3 respectively. The dipolar absorption efficiency of a metal sphere with radius $r \ll \lambda$ can be concisely described by the commonly used eqn: ${ }^{19}$

$$
Q_{a b s}=\frac{24 \pi r}{\lambda} \frac{\varepsilon_{m}^{3 / 2} \varepsilon^{\prime \prime}}{\left(\varepsilon^{\prime}+2 \varepsilon_{m}\right)^{2}+\varepsilon^{\prime \prime 2}}
$$

Where $\varepsilon_{\mathrm{m}}$ is the dielectric constant of the surrounding medium and $\varepsilon=\varepsilon^{\prime}+i \varepsilon^{\prime \prime}$ is the permittivity of the metal at wavelength $\lambda$. Eqn 1 is often misused as an exact description of the dipolar absorption efficiency of a sphere. However, a correction must be derived ${ }^{18}$ for the size dependence of the position of the absorption peak:

$$
\varepsilon_{p}^{\prime}=-2 \varepsilon_{m}-\frac{12}{5} \frac{4 \pi^{2} r^{2} \varepsilon_{m}^{2}}{\lambda_{p}^{2}}
$$

where $\varepsilon_{p}^{\prime}$ gives the value of the real part of the dielectric function at the peak position. Hence, by substituting this condition for the absorption peak into (1) the absorption efficiency becomes:

$$
Q_{a b s}=\frac{24 \pi r}{\lambda} \frac{\varepsilon_{m}^{3 / 2} \varepsilon^{\prime \prime}}{\varepsilon^{\prime \prime 2}+\left(\varepsilon^{\prime}+2 \varepsilon_{m}+\frac{48 \pi^{2} r^{2} \varepsilon_{m}^{2}}{5 \lambda^{2}}\right)^{2}}
$$

The absorption efficiency of the dipole resonance in a sphere is now dependent on particle size and is a function of $\varepsilon^{\prime}$, the size parameter $2 \pi r \varepsilon_{m}{ }^{1 / 2} / \lambda$ and $\varepsilon^{\prime \prime}$. The position of the plasmon resonance $\left(\lambda_{p}\right)$ occurs when the part of Eqn 3 in brackets is zero. Counter-intuitively, it is now clear that the absorption efficiency of the sphere is inversely proportional to losses in the metal $\left(\varepsilon^{\prime \prime}\right)$. This can be best explained by saying that losses in the metal damp the surface plasmon. Conversely, decreased loss in the metal leads to increased energy absorption by the sphere. Recently, we showed ${ }^{20}$ that the dependence of $\left(\lambda_{p}\right)$ on sphere radius is inversely proportional to the negative derivative of the real part of the dielectric constant with respect to wavelength.

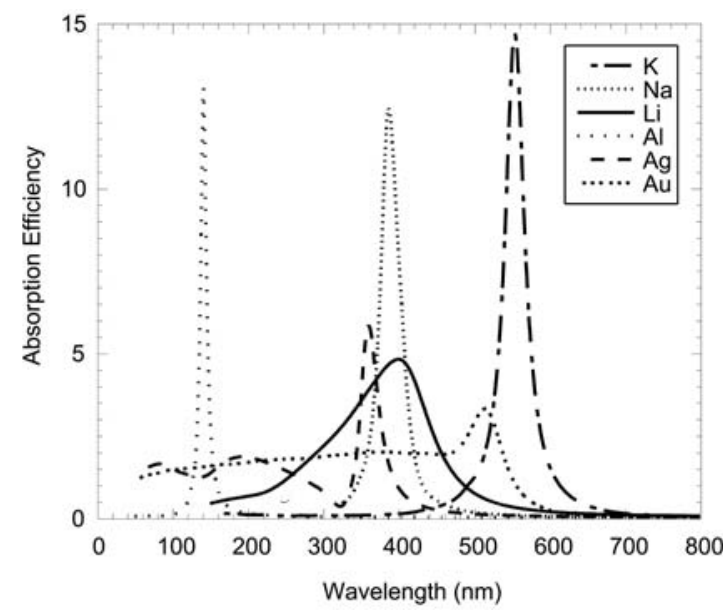

Fig 1 Absorption spectra for select alkali and noble metal spheres with radius detailed in Table 1.

Table 1 The optimized absorption efficiencies for select alkali and noble metals. $\lambda_{p}$ is the wavelength position of the plasmon peak.

\begin{tabular}{ccccc}
\hline Element & $\mathbf{Q}_{\mathbf{a b s}}$ & $\begin{array}{c}\mathbf{r} \\
(\mathbf{n m})\end{array}$ & $\begin{array}{c}\boldsymbol{\lambda}_{\mathbf{p}} \\
(\boldsymbol{\mu} \mathbf{m})\end{array}$ & $\begin{array}{c}\boldsymbol{\delta} \boldsymbol{\varepsilon}^{\prime} / \boldsymbol{\delta} \boldsymbol{\lambda} \\
\left(\boldsymbol{\mu} \mathbf{m}^{-1}\right)\end{array}$ \\
\hline $\mathrm{Li}$ & 4.8 & 30 & 0.401 & -17.72 \\
$\mathrm{Na}$ & 12.4 & 16 & 0.387 & -17.07 \\
$\mathrm{Al}$ & 13.0 & 6 & 0.143 & -45.41 \\
$\mathrm{~K}$ & 14.7 & 21 & 0.555 & -10.69 \\
$\mathrm{Ag}$ & 5.8 & 23 & 0.361 & -52.81 \\
$\mathrm{Au}$ & 3.3 & 49 & 0.516 & -66.48
\end{tabular}

\section{Resolution of alkali and noble metal superlenses}

Bulk dielectric constants of Weaver and Frederikse $^{16}$ are used again to calculate the ability of a thin film of $\mathrm{Al}, \mathrm{Na}, \mathrm{K}, \mathrm{Ag}$ and $\mathrm{Au}$ with thickness $d=40 \mathrm{~nm}$ and permittivity $\varepsilon=\varepsilon^{\prime}+i \varepsilon^{\prime \prime}$ to reconstruct the image formed by a grating with period $P$. The grating is placed at $z=0$ with infinite length in the $y$ direction. If the permittivity of the film is appropriately matched to the permittivity of the surrounding medium then the image will be constructed at a distance $z=2 d=80 \mathrm{~nm}$ from the object. For the sake of simplicity, the film is placed half-way 
between the image and the object (Fig 2); although it has been shown that there is merit in placing the lens such that it is touching the object plane. ${ }^{21}$

The criteria for a resolved image was $10 \%$ visibility at the image plane $(z=80 \mathrm{~nm})$ :

$$
V=\frac{I_{\max }-I_{\min }}{I_{\max }+I_{\min }}
$$

Where $I_{\min }$ occurs at $x=-P, 0, P\left(\right.$ Fig 2) and $I_{\max }$ occurs at $x=-P / 2, P / 2$ due to grating periodicity.

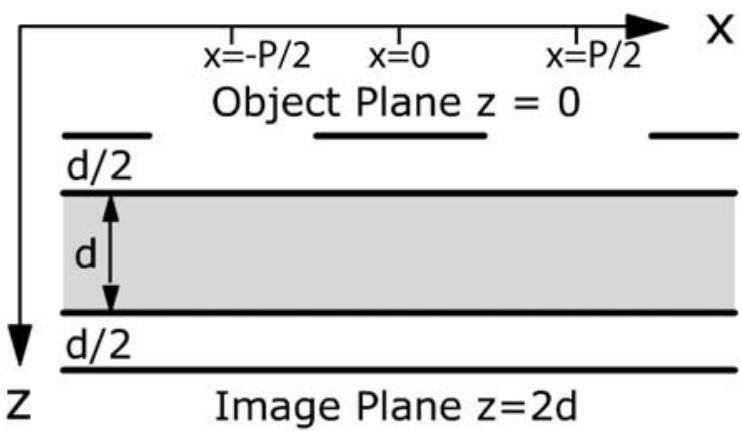

Fig. 2 Theoretical setup with the lens starting at $z=20 \mathrm{~nm}$ and ending at $z=60 \mathrm{~nm}$.
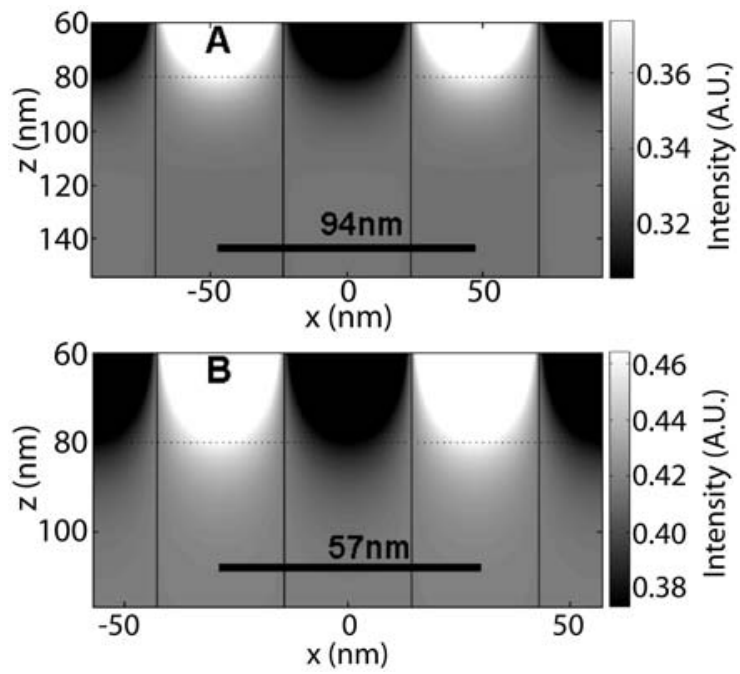

Fig. 3 The absolute value of the image of a grating with a) $P=94 \mathrm{~nm}$ with a silver lens and b) $P=57 \mathrm{~nm}$ with a potassium lens. The image plane lies at $z=$ $80 \mathrm{~nm}$ and is marked with a dashed line in both cases. Solid vertical lines indicate the $x$ position of the object.

Although Ruppin and Kempa showed ${ }^{22}$ that non-local effects affect the resolution of $\mathrm{Ag}$ and $\mathrm{K}$ films, we shall use Pendry's derivation of image transmission through a thin film ${ }^{9}$. Reconstruction of the near-field requires the excitation of surface modes on the film; hence, we assume p-polarized light. Wavelengths and permittivities used for the calculation are documented in Table 2 and images of the extent of the field beyond the lens $(z>60 \mathrm{~nm})$ are shown in Fig 3. Note that, wavlengths in Table 2 differ from those in Table 1 because the latter correspond to the surface plasmons on a sphere, or where the real part of the dielectric function is around -2 . The minimal resolvable feature in $x\left(x_{\min }\right)$ and the resolution enhancement $\quad\left(R_{E}=\right.$ $\left.\lambda / x_{\min }\right)$ can also be determined approximately by: $:^{21,23}$

$$
x_{\min }=-\frac{2 \pi d}{\ln (\Delta \varepsilon / 2)}
$$

where $\Delta \varepsilon=\varepsilon^{\prime \prime}$, and we assume, for the purpose of determining the resolution, that the real part of the permittivity is -1 and so only $\varepsilon^{\prime \prime}$ contributes to loss of image fidelity. For K, $x_{\min } \sim 80 \mathrm{~nm}$, in Fig 3B we demonstrate a $\mathrm{K}$ film resolving a $29 \mathrm{~nm}$ feature. For silver, $x_{\min }=180$ nm, Fig 3A shows reconstruction of the image of a $47 \mathrm{~nm}$ feature. The gold film exhibits negative resolution enhancement. For $\varepsilon^{\prime \prime}>1$, any thickness film will reduce image fidelity. The difference between the resolution predicted by Fig 3 and Eqn 5 is due to the periodicity of the structure in Fig 3; only a single Fourier component of the object need be reconstructed. Of greater significance is the resolution enhancement that a potassium film provides (5.47x) compared to a silver film (only 1.86x).

Table 2 Wavelengths at which the real part of the permittivity of the metal is approximately -1 .

\begin{tabular}{cccccc}
\hline Element & $\lambda(\boldsymbol{\mu} \mathbf{m})$ & $\boldsymbol{\varepsilon}^{\prime \prime}$ & $\begin{array}{c}\boldsymbol{\delta} \boldsymbol{\varepsilon}^{\prime} / \boldsymbol{\delta} \boldsymbol{\lambda} \\
\left(\boldsymbol{\mu} \mathbf{m}^{-1}\right)\end{array}$ & $\begin{array}{c}\boldsymbol{x}_{\min } \\
(\mathbf{n m})\end{array}$ & $\boldsymbol{R}_{\boldsymbol{E}}$ \\
\hline $\mathrm{Al}$ & 0.114 & 0.074 & -36.73 & 76 & 1.5 \\
$\mathrm{Na}$ & 0.311 & 0.097 & -11.70 & 83 & 3.7 \\
$\mathrm{~K}$ & 0.436 & 0.086 & -11.34 & 80 & 5.5 \\
$\mathrm{Ag}$ & 0.341 & 0.497 & -75.83 & 180 & 1.9 \\
$\mathrm{Au}$ & 0.481 & 3.53 & -45.00 & -440 & -1.1
\end{tabular}

If now the derivative of the permittivity when the real part is -1 is taken into consideration (i.e. $\Delta \varepsilon=\Delta \varepsilon^{\prime}+\varepsilon^{\prime \prime}$ ), and we assume that our light source is $5 \mathrm{~nm}$ from the ideal (ideal is $341 \mathrm{~nm}$ for $\mathrm{Ag}$ ), the resolution enhancement for $\mathrm{Ag}$ becomes negligible. The permittivity of $\mathrm{Ag}$ at $346 \mathrm{~nm}$ is $-1.35+0.53 \mathrm{i}$ resulting in $\Delta \varepsilon=0.88$, whereas illuminating a potassium system (ideal 
$436 \mathrm{~nm}$ ) with light of wavelength $441 \mathrm{~nm}$, $\varepsilon=-1.05+0.9 \mathrm{i}$, and hence $\Delta \varepsilon=0.14$.

Deviations from the ideal condition of $\varepsilon=-1$ adversely affect image reconstruction. Choosing a metal with small absolute permittivity gradient reduces the effects of a non-perfect light source. For $\mathrm{Au}, \mathrm{Ag}$ and $\mathrm{Al}$ the range of wavelengths at which the permittivity is approximately -1 is very small. $\mathrm{K}$ and $\mathrm{Na}$ have very low permittivity gradients compared to the other metals, resulting in a large range of wavelengths.

\section{Conclusions}

BHCOAT, an implementation of Mie theory has been used to calculate the absorption efficiency of $\mathrm{Al}, \mathrm{Ag}, \mathrm{Au}, \mathrm{Na}, \mathrm{K}$ and $\mathrm{Li}$ nanospheres. Also, a formula has been constructed that quite accurately describes the size dependence of the dipole resonance in a sphere. Potassium has the highest $Q_{a b s}$ of 14.7 as a direct result of minimal imaginary permittivity. Using this as a basis for determining which metals have the least losses, a comparison of the imaging properties of thin films of the aforementioned metals has been presented. The problem with losses in silver films can be overcome by using an alternative metal, namely potassium, which can resolve a grating with period $57 \mathrm{~nm}$ when $\mathrm{d}=40 \mathrm{~nm}$. The permittivity of potassium is also within $5 \%$ of 1 over a $9 \mathrm{~nm}$ range of wavelengths compared to silver's $1.3 \mathrm{~nm}$.

\section{Acknowledgements}

This work was supported by the Australian Research Council, and the University of Technology, Sydney. Computing resources were provided by the Australian Centre for Advanced Computing and Communication (ac3) in New South Wales and the National Facility at the Australian Partnership for Advanced Computing (APAC).

\section{References}

[1] M. Cortie, et al., Proc. SPIE 5649, 565 (2005).

[2] J. Shan, et al., Langmuir 22, 794 (2006).

[3] D. Pissuwan, et al., Trends. Biotechnol. 24, 62 (2006).

[4] D. P. O'Neal, et al., Canc. Lett. 209, 171 (2004).

[5] S. Q. Sun, et al., Nano Lett. 6, 345 (2006).

[6] K. L. Kelly, et al., J. Phys. Chem. B 107, 668 (2003).

[7] N. Harris, et al., J. Phys. Chem. B 110, 10701 (2006).

[8] V. G. Veselago, Sov. Phys. Uspek. 10, 509 (1968).

[9] J. B. Pendry, Phys. Rev. Lett. 85, 3966 (2000).
[10] R. J. Blaikie, et al., Microelectron. Eng. 83, 723 (2006).

[11] N. Fang, et al., Science 308, 534 (2005).

[12] D. O. S. Melville, et al., Opt. Exp. 13, 2127 (2005).

[13] D. O. S. Melville, et al., Curr. Appl Phys. 6, 415 (2006).

[14] J. T. Shen, et al., Appl. Phys. Lett. 80, 3286 (2002).

[15] J. B. Pendry, Contemp. Phys 45, 191 (2004).

[16] J. H. Weaver, et al., Optical properties of selected elements 82 ed. (CRC Press, Boca Raton, FL, 2001).

[17] G. Mie, Ann. Phys. 25, 377 (1908).

[18] C. F. Bohren, et al., Absorption and scattering of light by small particles (Wiley, Weinheim, 2004).

[19] U. Kreibig, et al., Optical properties of metal clusters (Springer-Verlag, Berlin Heidelberg, 1995).

[20] M. G. Blaber, et al., in 2006 ICONN Proceedings, Brisbane, Australia, IEEE, 2006, Vol. Submitted.

[21] V. A. Podolskiy, et al., Appl. Phys. Lett. 87, 231113 (2005).

[22] R. Ruppin, et al., Phys. Rev. B 72,153105 (2005).

[23] D. R. Smith, et al., Appl. Phys. Lett. 82, 1506 (2003). 